\title{
Bayesian-Estimation-Algorithm-Based Gas Detection Modules
}

\author{
Kuo-Lan Su*, Yi-Lin Liao', Sheng-Wen Shiau ${ }^{1}$ and Jr-Hung Guo ${ }^{1}$ \\ Department of Electrical Engineering, National Yunlin University of Science \& Technology \\ Douliou, Yunlin, Taiwan \\ ${ }^{1}$ Graduate School of Engineering Science and Technology, National Yunlin University of Science \\ \& Technology, Douliou, Yunlin, Taiwan
}

(Received November 5, 2012; accepted February 4, 2013)

Key words: intelligent building system, Bayesian estimation algorithm, Holtek microchip

In this study, we develop two types of gas detection module using multiple sensors and applied them to the intelligent building system. Bayesian estimation algorithm is applied in the competitive gas detection module and complementary gas detection module, and the proposed algorithm is implemented for various gas sensor combination methods. In the competitive gas detection module, we use two gas sensors to improve the accuracy of the proposed algorithm. In the complementary gas detection module, we use an $\mathrm{NH}_{3}$ sensor, an air pollution sensor, an alcohol sensor, a HS sensor, a smoke sensor, a CO sensor, an LPG sensor, and a natural gas sensor. The module classifies various unknown gases using Bayesian estimation algorithm. The controller of the two gas detection modules is a Holtek microchip. The modules can communicate with the supervised computer via a wired series interface or a wireless RF interface and alarm users using the voice module. Finally, we present some experimental results to measure known and unknown gases using the two gas detection modules.

\section{Introduction}

An intelligent building system (IBS) is the integration of various services and contains security system, building heating, heat ventilation and air-conditioning (HVAC) technologies, computer system, telecommunication, and Internet. The most important aspect of the intelligent building is the security system. How do we build a safe and useful security system? It is an important problem for the intelligent building. In the security system, redundant and complementary information results can enhance system reliability and certainty using multisensor fusion algorithms, and such system uses a module-based structure to make it easy to operate.

Developing module-based security devices is an important work for human life. In general, it is very important to detect harmful gases. We want to develop an intelligent

*Corresponding author: e-mail: sukl@yuntech.edu.tw 
gas detection module using multiple gas sensors. The module enhances the detection accuracy using a multisensor fusion algorithm and decreases the probability of a dangerous event. We design two gas detection modules using competitive sensors or complementary sensors, and develop wired and wireless communication interfaces.

A gas detection module is an instrument comprising an array of chemical sensors to be combined in a board, and a pattern recognition system is embedded in the controller to classify the gas. ${ }^{(1)}$ The gas detection system plays an important role in many fields, for example, environmental protection, production safety, food surveillance, intelligent transport system, and life support system for manned spaceship.(2) To classify a single gas in the environment, various pattern recognition technologies have been applied to the gas detection system. The most important methods include principal component analysis (PCA), cluster analysis (CA), and discriminate function analysis (DFA). ${ }^{(3)}$ Meanwhile, quantitative data processing techniques such as multiple linear regression (MLR) and artificial neural networks (ANNs) are also used to quantify the gas concentration. ${ }^{(4)}$

\section{System Architecture}

The system architecture of the gas detection module is shown in Fig. 1. The module contains some gas sensors, a voice module, a supervised computer, a wired series interface, and a wireless RF interface. The controller of the gas detection modules is a Holtek microchip. The signals of the gas sensors are transmitted to the controller of the gas module through preprocessing circuits and to the supervised computer via a wired series interface or a wireless RF interface.

In the gas leakage detection module (competitive gas module), we use two identical gas sensors to detect gas leakage in the chamber and Bayesian estimation algorithm to decide if the event happens or not. The prototype of the module is shown in Fig. 2(a). The right side of Fig. 2(a) shows two types of competitive gas detection module. One uses a wired series interface to communicate with the supervised computer, and the other uses a wireless RF interface. The left side of Fig. 2(a) shows hardware devices of the wireless-based gas module. We set the same probability value for the two gas sensors. Then, we set two threshold values to decide the output status. One is low threshold value $\eta_{\mathrm{L}}$ for low density, and $\eta_{\mathrm{H}}$ is for high density. When the posterior probability value is calculated using the Bayesian estimation algorithm to be between $\eta_{\mathrm{L}}$ and $\eta_{\mathrm{H}}$, the module speaks in Chinese: "be careful of gas leakage". When the posterior probability value is over $\eta_{\mathrm{H}}$, the module speaks in Chinese: "gas leakage".

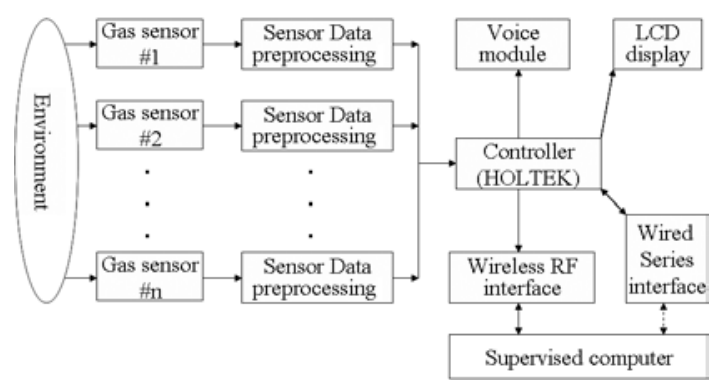

Fig. 1. Structure of gas detection module. 


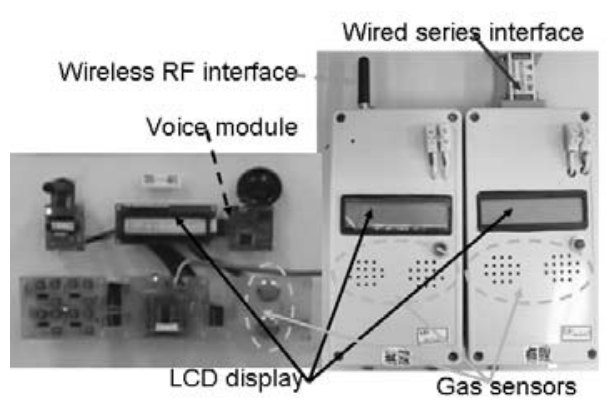

(a)

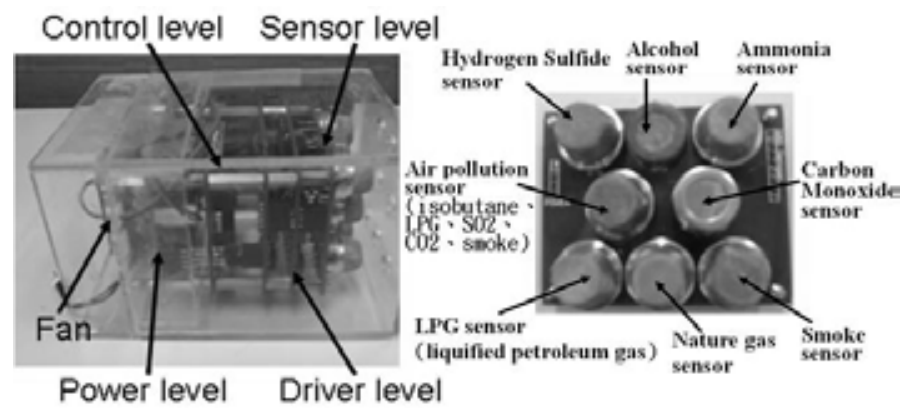

(b)

Fig. 2. Two gas detection modules: (a) competitive gas detection module and (b) complementary gas detection module.

In the complementary gas detection module, we use eight gas sensors embedded in a chamber to measure the unknown gas. Each gas sensor detects multiple gases, listed in Table 1. The HS133 and HS134 sensor elements have high sensitivity, long life, reliable stability, and good selectivity to low carbon monoxide concentration. The HS135 sensor element has prolonged stability and wide detection scope. It should be suitable for the detection of smoke, $\mathrm{SO}_{2}, \mathrm{CO}_{2}$, alcohol, etc. The sensor element HS129 can detect isobutene, alcohol, and hydrogen, and different gas concentrations will cause the resistance to change.

The prototype of the complementary gas detection module is shown in Fig. 2(b). The gas detection module contains four levels and a fan. There is a sensor level, a driver level, a control level, and a power level. The sensor level has eight gas sensors aligned in a board. The next level is preprocessing circuits for these gas sensors. The control level contains a Holtek microchip, a voice module, a wired series interface, and a wireless RF interface. The power level converts $\mathrm{AC}$ power to DC power, and provides DC power to each level. The fan decreases the temperature of the gas detection module and draws in wind from the sensor level.

\section{Algorithm Analysis}

Bayesian estimation provides formalism for multisensor fusion that allows sensory information to be combined according to the rules of probability theory. Uncertainty is represented in terms of conditional probabilities $P(Y \mid X)$. Each $P(Y \mid X)$ takes a value between 0 and 1. Bayesian estimation is based on the well-known Bayes' rule:

$$
P(Y \mid X)=\frac{P(X \mid Y) P(Y)}{P(X)} .
$$

Here, the "posterior probability" represents the belief value according to the hypothesis $Y$, giving the information represented by $X$. The redundant information from a fleet of $n$ sensors, $S_{1}$ through $S_{n}$, can be fused using the likelihood ratio formulation of Bayes' rule as follows: 
Table 1

Sensors of the complementary gas module.

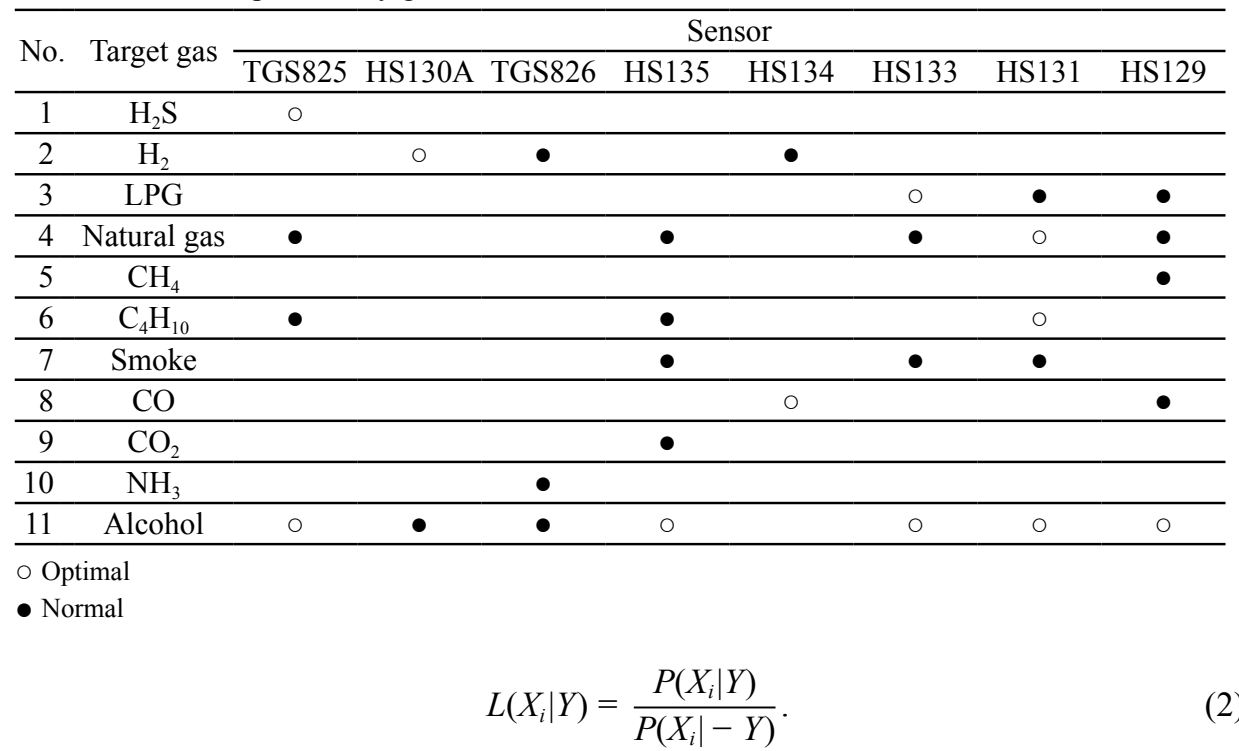

Assume that the operation of each gas sensor is independent of the gas detection module. The "posterior odds" on $Y$, giving the information $X_{1}, X_{2}, \ldots, X_{n}$ from the $n$ sensors, are given by the product.

$$
\begin{aligned}
& O\left(Y \mid X_{1}, X_{2}, \ldots, X_{n}\right)=O(Y) \prod_{i=1}^{n} \frac{P\left(X_{i} \mid Y\right)}{P\left(X_{i} \mid-Y\right)} \\
& O\left(Y \mid X_{1}, X_{2}, \ldots, X_{n}\right)=\frac{O\left(Y \mid X_{1}, X_{2}, \ldots, X_{n}\right)}{1+O\left(X_{1}, X_{2}, \ldots, X_{n}\right)}
\end{aligned}
$$

Equation (4) provided the posterior probability of the event. The sensory information can be assumed to be independent over time. We provide an example to explain how to use the proposed method in gas leakage detection. The event $Y$ uses two gas sensors, $X_{1}$ and $X_{2}$, on the module. We measure the density of gas leakage using the gas sensors $X_{1}$ and $X_{2}$, and discuss the case using two threshold values $\eta_{\mathrm{L}}=0.3$ and $\eta_{\mathrm{H}}=0.8$ for the gas detection module.

The gas sensor $X_{1}$ detects the gas density to be high, and the gas sensor $X_{2}$ detects the gas density to be low. We can find the sensory information $P\left(X_{1} \mid Y\right)=0.7$ and $P\left(X_{2} \mid Y\right)=$ 0.3 to be computed as the measurement voltage for the gas. The module calculates the posterior odds and posterior probability,

$$
O\left(Y \mid X_{1}, X_{2}\right)=\frac{0.7}{1-0.7} \frac{0.3}{1-0.3}=1, \quad P\left(Y \mid X_{1}, X_{2}\right)=\frac{1}{1+1}=0.5
$$


The posterior probability value is between $\eta_{\mathrm{L}}$ and $\eta_{\mathrm{H}}$. The gas detection module says "be careful of gas leakage".

\section{Experimental Results}

We present two gas detection modules to measure the known and unknown gases and implement the proposed algorithm to be right. In the competitive gas detection modules, we use two gas sensors to detect the gas leakage. The module transmits the detection results to the supervised computer via the wireless RF interface, shown in Fig. 3. We use one lighter to provide gas on the right-side gas sensor (\#1) of the module. The module speaks in Chinese: "be careful of gas leakage" to alarm the user, and the posterior probability is between $\eta_{\mathrm{L}}$ and $\eta_{\mathrm{H}}$. The experimental result is shown in Fig. 3(a). Then, we use two lighters to provide gas to both sides of the module. The module computes the posterior probability to be over $\eta_{\mathrm{H}}$, transmits the signal to the supervised computer, and speaks in Chinese: "gas leakage" for the condition [Fig. 3(b)].

We use the complementation gas detection module to detect a variety of unknown gases. First, the complementary gas detection module detects the gas leakage (unknown gas) of the building. We can see the output voltage of each gas sensor displaying the "Mean" on the monitor. Then, we can calculate the concentration of the unknown gas. First, we assume the unknown gas to be alcohol, and the ratios of these sensors \#1, \#2, \#3, \#4, \#6, $\# 7$, and \#8 to be about 0.4586 (TGS825), 0.1041 (HS130A), 0.1162 (TGS826), 0.3832 (HS135), 0.2403 (HS133), 0.46 (HS131), and 0.3209 (HS129), respectively. Gas sensor \#5 cannot detect the alcohol, and the ratio is zero. Then, we assume the unknown gas to be natural gas, and calculate the concentration ratios of these gas sensors. Sensors \#2, \#3, and \#5 cannot measure the unknown gas to be depleted according to Table 1. The decision output displays "gas" using the proposed algorithm, shown in Fig. 4(a). The probability of the gas $(0.9712)$ is higher than that of alcohol $(0.001)$.

Then, we use the module to detect another unknown gas (alcohol). We can see that the measurement values of these gas sensors are shown in the "Mean" of Fig. 4(b). First, we assume the unknown gas to be alcohol, and the ratios of sensors \#1, \#2, \#3, \#4, $\# 6$, \#7, and \#8 to be about $0.7647,0.9452,0.9376,0.7493,0.6245,0.56$, and 0.6798 ,

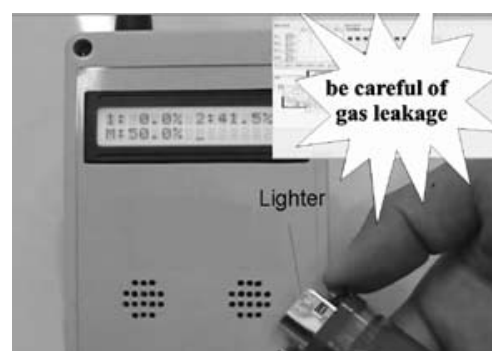

(a)

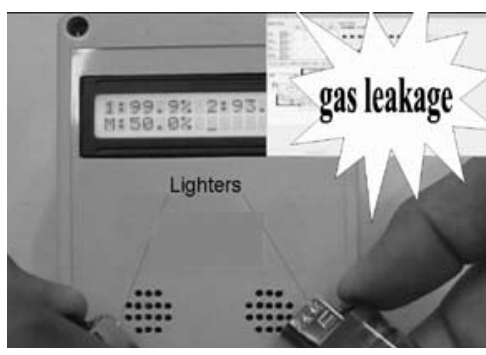

(b)

Fig. 3. Experimental results of the competitive gas module: (a) sensor \#1 detects gas leakage and (b) sensors \#1 and \#2 detect gas leakage. 


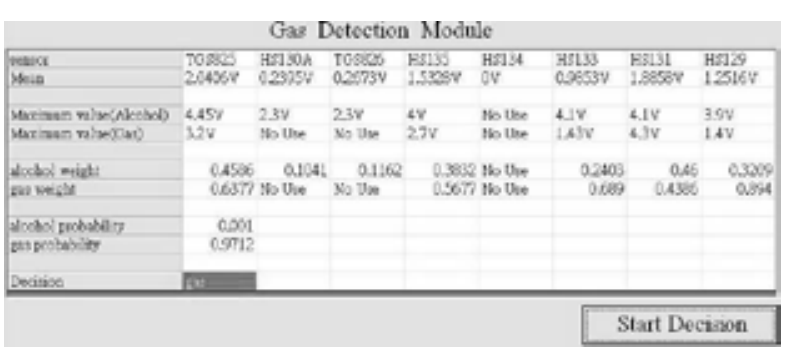

(a)

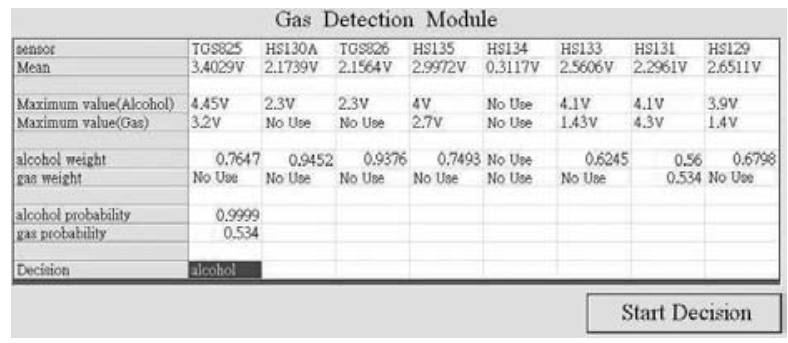

(b)

Fig. 4. Experimental results of the complementary gas module.

respectively. Then, we assume the unknown gas to be natural gas and calculate the concentration ratio of gas sensor \#5 to be 0.534 (HS131). Sensors \#2, \#3, and \#5 cannot measure if gas is depleted. The concentration ratios of sensors \#1, \#4, \#6, and \#7 are over 1, and are not reasonable for the unknown gas. The decision output displays "alcohol", shown in Fig. 4(b). The probability of alcohol (0.9999) is higher than that of the gas (0.534).

\section{Conclusions}

We have designed two gas detection modules using multiple chemical gas sensors for the security system of the intelligent building. The gas sensors of the detection modules are classified into two types. One module uses competitive gas sensors. The other uses complementary gas sensors. The gas detection module can measure known or unknown gases using Bayesian estimation algorithms and implement the proposed algorithm to be right, and transmits the detection results to the supervised computer via a wired or wireless interface. The controller of the two gas detection modules is a Holtek microchip and shows the measurement status in real-time using the voice module. In the experimental results, the complementary gas module can detect the unknown gas to be gas or alcohol using multiple gas sensors. In the future, we want to use multilevel fusion algorithms to improve the accuracy of the competitive and complementary gas detection modules.

\section{Acknowledgements}

This work was supported by the National Science Council of Taiwan (NSC 100-2221-E-224-018).

\section{References}

1 J. Temis and A. Selivanov: J. Vibroeng. 14 (2012) 483.

2 E. Scorsone, A. M. Pisanelli and K. C. Persaud: Sens. Actuators, B 116 (2006) 55.

3 A. Bermak and S. B. Belhouari: IEEE Trans. Instrum. Meas. 55 (2006) 787.

4 H. Zhang and M. Chang: Sens. Actuators, B 134 (2008) 332. 